\title{
Challenges in the Management of Mucopolysaccharidosis Type II (Hunter's Syndrome) in a Developing Country: a Case Report
}

\author{
Ibraheem Rasheeedah ${ }^{1}$, Oladele Patrick $^{1}$, AbdulAzeez Abdullateef ${ }^{\mathbf{1}}$, \\ Abdulkadri Mohammed ${ }^{1}$, Katibi Sherifat ${ }^{1}$, Ibraheem Gbadebo ${ }^{2}$
}

\begin{abstract}
BACKGROUND: Mucopolysaccharidosis type II (Hunter's syndrome) is an X-linked chromosomal storage disorder due to deficiency of the lysosomal enzyme iduronate-2-sulfatase with patients rarely living till adulthood. Failure to identify patients early could contribute to an increased morbidity as identified in this case report.

CASE DETAILS: An eight year old patient with Hunter's syndrome identified five years after disease onset with severe cardiovascular complications exemplifies the challenges faced in resource-limited countries towards making diagnosis and treatment of rare conditions. Elevated urinary glycosaminoglycans levels or a strong clinical suspicion of Hunter's syndrome, as identified in the index case, is a prerequisite for enzyme activity testing. Urinary mucopolysaccharide(MPS) level was $69.6 \mathrm{mg} / \mathrm{mmol}($ normal range is 0.0 $11.6 \mathrm{mg} / \mathrm{mmol}$ ), and the confirming MPS electrophoresis analysis showed elevated heparan sulphate in the urine sample. Enzyme activity testing, with absent or very low iduronate-2-sulfatase activity, is diagnostic. However, the scarce availability and high cost of these tests is another constraint in making a diagnosis.

CONCLUSION: Identification and management of mucopolysaccharidosis type II pose a problem in resource-constrained countries due to late presentation, lack of facility for diagnosis and treatment, cost and expertise required for the management.

KEYWORDS: Mucopolysaccharidosis, Hunter's syndrome, Mucopolysaccharide
\end{abstract}

DOI: http://dx.doi.org/10.4314/ejhs.v25i3.12

\section{INTRODUCTION}

Hunter's syndrome is a subset of Mucopolysaccharidosis, a group of metabolic disorders, caused by deficiency in the activity of lysosmal enzymes needed to breakdown long chain sugar molecules called glycosaminoglycans (1). It is a rare X-linked recessive disorder characterized by deficiency of iduronate-2-sulfatase, which catalyses a step in the catabolism of glycosaminoglycans (GAGs) resulting in accumulation of heparan and dermatan sulfate in many organs and tissues (2).

The syndrome occurs in all ethnic groups, but a higher incidence has been reported among the Jews in Israel (3). The incidence ranges from one case per 72,000 male live births in Northern Ireland to one case per 518,000 male live births in British Columbia (4-8).
The incidence of the disease is not known in Nigeria although two cases have been reported.

The disease is characterized by coarse facie, large and protruding tongue, infection and obstruction of the upper airways, hypoacusis, inguinal and umbilical hernia, joint contractures, skeletal abnormalities, hepatosplenomegaly, cardiac valve disease and and slowly progressive mental deterioration with behavioural alterations between the ages of 2 and 6 years $(4,9)$. Patients tend to be tall for their age until 4 or 5 years of age, when they begin to lag behind unaffected boy. Hunter's syndrome is often described as having two phenotypes, attenuated and severe, on the basis of life expectancy and the presence or absence of central nervous system complications $(4,10,11)$. Due to the insidious onset of the disease

\footnotetext{
${ }^{1}$ Department of Paediatrics and Child Health, University of Ilorin, Ilorin, Kwara, Nigeria

${ }^{2}$ Department of Surgery, University of Ilorin, Ilorin, Kwara, Nigeria

Corresponding Author: Ibraheem Rasheedah, Email: rasheedahbidmus@yahoo.com
} 
with the presence of recurrent ear infections, identification may become possible only when the coarse features appear usually at the age of two to three years (9).

We report a patient with Hunter's syndrome who was identified late with severe cardiovascular complications. Also, highlighted are the compounding challenges in the management of this condition in a resource-constrained environment like Nigeria.

\section{CASE REPORT}

An 8 year old boy admitted via respiratory clinic due to swollen legs and abdomen of 8 months duration and progressive difficulty $\mathrm{n}$ ibreathing of 4 months. His parents noticed an abnormal facial appearance when he was about 3 years old, described as prominent forehead, protruding eyes and enlarged jaws with thickened skin. He had presented at the Ear, Nose and Throat clinic with complaints of snoring and recurrent ear discharge with resultant hearing impairment. He was on regular follow-up at the ENT outpatient clinic for 5 years without identification of the background dysmorphic features, and was subsequently referred to the paediatric pulmonology clinic due to the progressive difficulty in breathing. There had been a regression in language development as he could no longer communicate in sentences, which he was previously able to do. No other significant past medical history was identified.

He was exclusively breastfed for 6 months and then complementary feeds added to his diet. Breastfeeding was stopped at the age 14 months.

The eldest of 2 children, his sibling was a 5 year old healthy boy, and there was no positive family history of a similar clinical condition. The patient started pre-nursery school at the age of 2 years, but had to be withdrawn 2 years later due to recurrent illnesses, requiring frequent hospital visits. He lived with his paternal grandmother following the separation of his parents when he was 4 years old. The father was an artisan and the mother a petty trader.

Examination revealed a big head with frontal bossing and caput quadratum, low set ears, depressed nasal bridge, up-turned nose, enlarged jaws, protruding tongue, short neck and short stubby digits with papular lesions on the upper trunk (Figures 1 and 2). He had an inspiratory stridor, was centrally cyanosed, afebrile, not pale, anicteric and had pitting oedema up to the thigh and sacrum. His height was $98 \mathrm{~cm}(81 \%$ of expected). He had joint stiffness with the fore-arms slightly flexed at elbow joints.

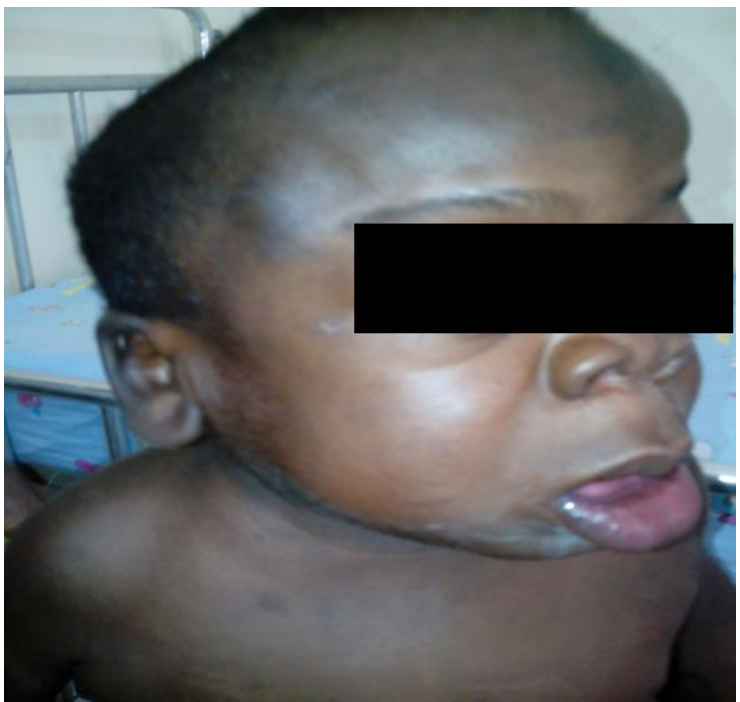

Figure1: Dysmorphic facial appearance

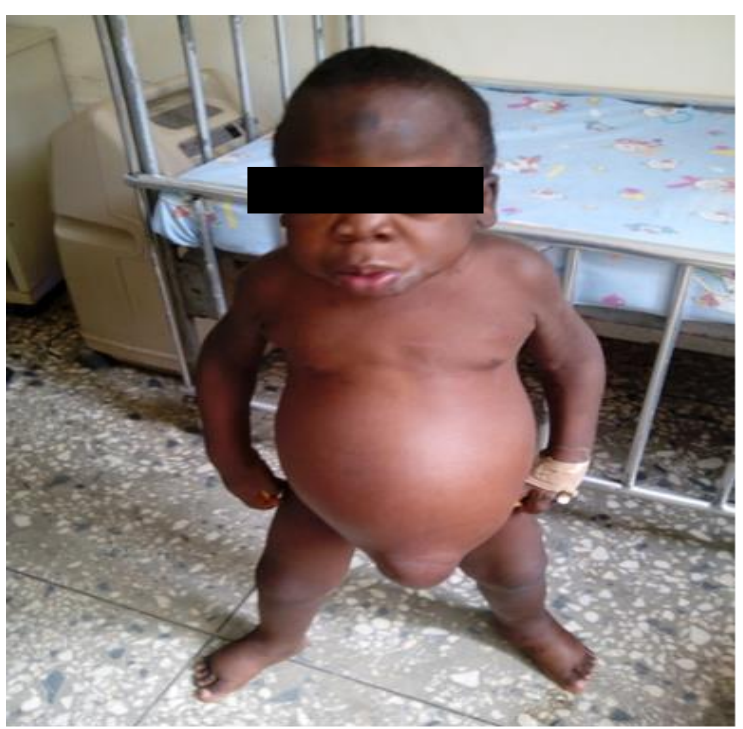

Figure 2: Slightly flexed forearms at elbow joints and gross abdominal distension with umbilical hernia

He had tachycardia, hyperactive precordium and apex beat in the $5^{\text {th }}$ intercostals space $2 \mathrm{~cm}$ lateral to midclavicular line. There was grade 3 pansystolic murmur loudest at the apex, radiating to the axilla. He was tachypneic and had transmitted sounds in the chest. The abdomen was distended with an umbilical hernia (Figure 2); there was tender hepatosplenomegaly and ascites demonstrable by fluid thrill.

Full blood count revealed a WBC of $4.1 \times 10^{9} / \mathrm{L}$ with 79\% lymphocytes, $16 \%$ neutrophils and anisopoikilocytosis, macrocytosis, hypochromia and target cells on blood film. Haemoglobin genotype was 
AA identified using cellulose acetate $\mathrm{Hb}$ electrophoresis. Serum electrolytes were essentially normal. Lateral skull X-ray showed a widened Jshaped sella turcica (Figure 3). There was a reduction in the vertical height, posterior displacement and anterior beaking of L3 vertebra and reduction of L2L3 disc space (Figure 4). Echocardiography showed mitral valve prolapse with severe regurgitation, moderate pericardial effusion and severe pulmonary hypertension.

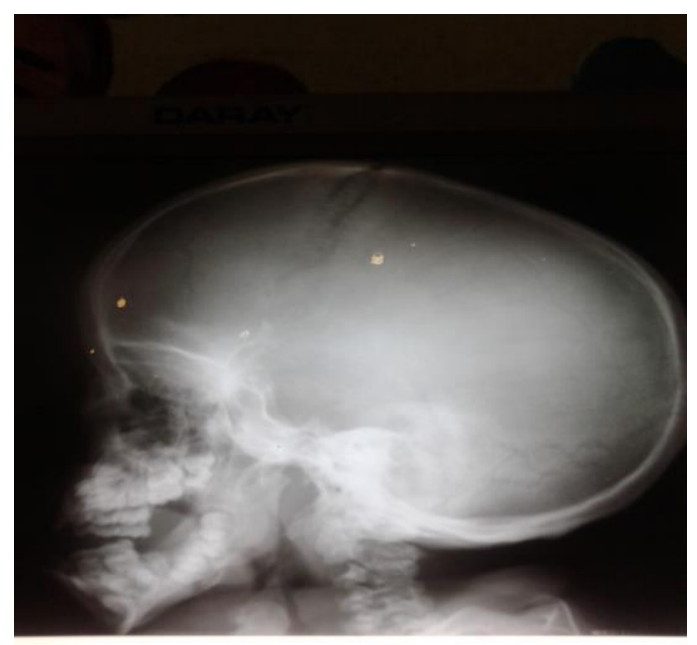

Figure3: Lateral skull X-ray showing a widened Jshaped sella turcica

Urinary creatinine was $1.25 \mathrm{mmol} / \mathrm{L}$, urate: creatinine ratio $1.46(0.13 \quad-\quad 0.94)$ and urinary mucopolysaccharide of $69.6 \mathrm{mg} / \mathrm{mmol}$ (normal range 0.0 - 11.6). The mucopolysaccharide (MPS) dimethyl methylene blue (DMB) screening was positive and the confirming MPS electrophoresis analysis showed elevated heparan sulphate in the urine sample.

$\mathrm{He}$ was examined during admission by the cardiologist, neurologist, otorhinolaryngologist, ophthalmologist, dermatologist, orthopaedic surgeon and physiotherapist. The child was on diuretics, captopril and also sildenafil for the severe pulmonary hypertension. Abdominal paracentesis was done twice and serial echocardiograms was done to monitor the size of pericardial effusion and pulmonary pressure. Enzyme replacement therapy could not be commenced because of financial difficulties and non-availability in this environment. He was discharged after about 3 weeks of admission following immense pressure by the parents while he was relatively stable. He was never returned for follow-up despite phone calls to the parents. He died at home a month after discharge from the hospital, and a post-mortem could not be performed.

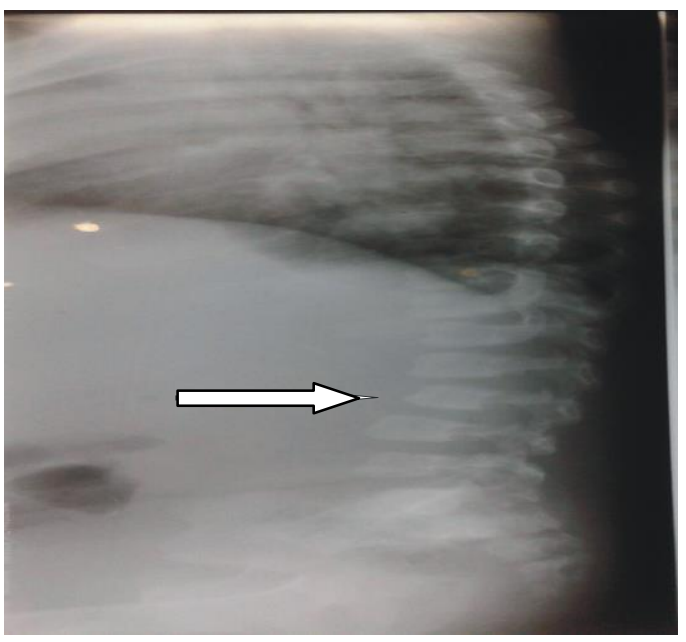

Figure 4: Anterior beaking of L3 vertebra (white arrow) with reduction in the vertical height and posterior displacement

\section{DISCUSSION}

Hunter's syndrome can be extremely difficult to diagnose before irreversible organ and tissue damage occurrs because of insidious onset and overlap in signs and symptoms with common childhood complaints (10). The challenges faced in resource poor countries in making diagnosis and treatment of rare conditions has been exemplified with the case reported.

Our patient presented with typical features of Hunter's syndrome as highlighted in earlier case reports $(1,2)$. Ogunbiyi et al (2) made the diagnosis mainly based on physical and radiological features, while in addition to these parameters, Chinawa et al (1) did a urine chemistry which showed normal urinary mucopolysaccharide levels. In contrast, the index patient was identified with a combination of physical and radiological features as well as urinary features of highly elevated urinary mucopolysaccharide with a positive mucopolysaccharide (MPS) dimethyl methylene blue (DMB) screen and the confirming MPS electrophoresis analysis which showed elevated heparan sulphate. The index patient and the patients in the earlier reports had similar physical and radiological features. Thus, an initial screening based on the physical appearance and radiological features will aid the diagnosis when Hunter's syndrome is suspected.

In order to help optimize patient outcomes, early identification, diagnosis and referral are critical. Although not curative, early treatment with enzyme replacement therapy before irreversible organ damage 
occurs may result in the greatest clinical benefit (10). The index patient was identified late already manifesting features of cardiac damage and other complications. The patient also presented late after being referred to a paediatrician due to financial constraints. Ignorance, poor health seeking behaviour and poverty are among the reasons for late presentation in developing countries.

The level of urinary GAGs is increased in patients with any Mucopolysaccharidosis; so the detection of excessive urinary GAG excretion is generally the first diagnostic approach although patients with a family history of Mucopolysaccharidosis II should proceed directly to enzyme activity assays and/or molecular genetic analyses (11). When urinary GAG levels are elevated, or there is a strong clinical suspicion of Hunter's syndrome as was noted in the index case, enzyme activity testing should be conducted. Unavailability of facilities for proper investigation also contributed to the difficulty in the management of the index case as the urinary GAG had to be done abroad. Absent or very low iduronate-2-sulfatase activity is diagnostic (10); however, the cost of doing this test is very high in resource-constrained countries or where the facility is not even available.

Management is multidisciplinary including cardiology, neurosurgery, ophthalmology, orthopaedics, otorhinolaryngology and pulmonology. Supportive services such as physiotherapy, speech therapy, audiology, dentistry and behavioural therapy are also involved (10). Patients and caregivers are often overwhelmed with the number of paediatric subspecialties that are involved in care as was the case in this instance. This could have contributed to the refusal for readmission.

In conclusion, identification and management of mucopolysaccharidosis type II in affected patients pose a problem in resource-constrained countries due to late identification and presentation, lack of facilities for diagnosis and treatment, as well as the cost and the expertise required for the management. A high index of suspicion based on the clinical features is needed to aid in early diagnosis before development of diseaserelated complications.

\section{REFERENCES}

1. Chinawa J, Adimora G, Obu H, Tagbo B, Ujunwa F, Onubogu I. Clinical Presentation of Mucopolysaccharidosis Type II (Hunter's Syndrome). Ann Med Health Sci Res. 2012;2(1):87-90.

2. Ogunbiyi A, Adeyinka AO, Ogah SO, Baiyeroju AM. Hunter syndrome- case report and literature review. WAJM. 2006;25(2):3.

3. Lopez-marin L, Gutiérrez-solana LG, Azuara LA, Heras RS, Rodriguez AD, Extremera VC. Detection by urinary GAG testing of mucopolysaccharidosis type II in an at-risk Spanish population. JIMD Rep. 2013 2;10:61-68.

4. Martin R, Beck M, Eng C, et al. Recognition and diagnosis of mucopolysaccharidosis II (Hunter syndrome). Pediatrics. 2008;121:377-386.

5. Gajula P, Ramalingam K, Bhadrashetty D. A rare case of mucopolysaccharidosis: Hunter syndrome. J Nat Sci Biol Med. 2012;3(1):97-100.

6. Lin $\mathrm{H}-\mathrm{Y}$, Lin S-P, Chuang C-K, et al. Incidence of the mucopolysaccharidoses in Taiwan, 19842004. Am J Med Genet Part A. 2009;149A(5):960-4.

7. Martínez-Quintana E, Rodríguez-González F. Mucopolysaccharidosis Type II and the G374sp Mutation. Mol Syndromol. 2013;4(4):203-6.

8. Nelson J, Crowhurst J, Carey B, Greed L. Review article incidence of the mucopolysaccharidoses in Western Australia. Am J Med Genet. 2003;313:310-313.

9. Wraith JE, Beck M, Giugliani R, Clarke J, Martin $\mathrm{R}$, Muenzer J. Initial report from the Hunter outcome survey. Genet Med. 2008;10:508-516.

10. Burton BK, Giugliani R. Diagnosing Hunter syndrome in pediatric practice: practical considerations and common pitfalls. Eur $J$ Pediatr. 2012;171:631-639.

11. Scarpa M, Almássy Z, Beck M, Bodamer O, Bruce I, De Meirleir L, et al. Mucopolysaccharidosis type II: European recommendations for the diagnosis and multidisciplinary management of a rare disease. Orphanet J Rare Dis. 2011;6:72. 